\title{
TWO-PERSON RED-AND-BLACK GAMES WITH BET-DEPENDENT WIN PROBABILITY FUNCTIONS
}

\author{
MAY-RU CHEN *** AND \\ SHOOU-REN HSIAU, ${ }^{* * *}$ National Changhua University of Education
}

\begin{abstract}
In this paper a two-person red-and-black game is investigated. We suppose that, at every stage of the game, player I's win probability, $f$, is a function of the ratio of his bet to the sum of both players' bets. Two results are given: (i) if $f$ is convex then a bold strategy is optimal for player I when player II plays timidly; and (ii) if $f$ satisfies $f(s) f(t) \leq f(s t)$ then a timid strategy is optimal for player II when player I plays boldly. These two results extend two formulations of red-and-black games proposed by Pontiggia (2005), and also provide a sufficient condition to ensure that the profile (bold, timid) is the unique Nash equilibrium for players I and II. Finally, we give a counterexample to Pontiggia's conjecture about a proportional $N$-person red-and-black game.
\end{abstract}

Keywords: Red-and-black game; bold strategy; timid strategy; convex function; Nash equilibrium

2000 Mathematics Subject Classification: Primary 91A15

Secondary 91A05; $91 \mathrm{~A} 06$

\section{Introduction}

The red-and-black gambling problem, which has taken its name from the game of roulette, has interested probabilists for quite some time. The discrete version of this gambling problem can be described as follows. A player begins with a positive integer fortune and wants to reach a goal $M$ by betting, at each stage of the game, an integer amount not greater than his current fortune. If the player's current fortune is $x$ and he stakes an amount $a$, then his next fortune will be $x+a$ with a certain fixed probability $w(0<w<1)$, and will be $x-a$ with probability $1-w$. Betting repeats until the goal $M$ is reached or the player goes broke. The problem is to find a strategy which maximizes the probability of the player reaching the goal.

Dubins and Savage [1, pp. 83-89] showed that, in the subfair case (i.e. $w \leq \frac{1}{2}$ ), an optimal strategy is bold play, which corresponds to always betting either the entire current fortune or just enough to reach the goal, whichever is smaller. This seems intuitively reasonable, in that a shorter game seems to give a better chance to the subfair player since he will surely lose in the long run. In the superfair case (i.e. $w \geq \frac{1}{2}$ ), Ross [4] proved that it is optimal for the player to bet timidly, that is, always to stake one unit of his current fortune at every opportunity. Intuitively, if the player is superfair, for him it is better to prolong the game.

Received 7 June 2006; revision received 21 August 2006.

* Postal address: Department of Mathematics, National Changhua University of Education, No. 1 Jin-De Rd., Changhua 500, Taiwan, R. O. C.

** Email address: mayru@ms29.url.com.tw

*** Email address: srhsiau@cc.ncue.edu.tw 
A two-person red-and-black game was introduced by Secchi [5]. In this game, one player wants to maximize the probability of reaching a goal while the second player chooses his actions to keep the first player from reaching the goal. Secchi provided an appropriate extension of bold play as an $\varepsilon$-optimal strategy for the player in the subfair case, and an extension of timid play as an $\varepsilon$-optimal strategy for the player in the superfair case.

Pontiggia [3] proposed two different formulations of two-person red-and-black stochastic games in which the players' win probabilities are not fixed, but change at each stage of the game. One formulation is called a weighted two-person red-and-black game, in which a player's win probability is the product of a weight and the ratio of his bet to the sum of the two bets. The other formulation is called a proportional two-person red-and-black game, in which the players' win probabilities are proportional to their bets. For both games Pontiggia showed that bold play is optimal for the player in the subfair case and timid play is optimal for the player in the superfair case.

Pontiggia also proposed an $N$-person red-and-black game with bet-dependent win probabilities, called a proportional $N$-person red-and-black game. She showed that if this game is fair to every player, then any profile of strategies is optimal. For games which are not fair to all players she made the following conjecture: at each stage of the proportional $N$-person red-and-black game, it is Nash for each player to play boldly if he is subfair and to play timidly otherwise. We will propose a counterexample to this conjecture in Section 3.

The organization of this paper is as follows. In Section 2 we consider a two-person red-andblack game in which at each stage the win probability of player I is a function of the ratio of his bet to the sum of the two bets. We first show that if the win probability function is convex, then an optimal strategy for player I is bold play when player II plays timidly. Next we show that if the win probability function $f$ satisfies $f(s) f(t) \leq f(s t)$, then it is optimal for player II to bet timidly when player I plays boldly. Several examples are discussed as applications of these two results. In particular, Pontiggia's two formulations of the two-person red-and-black game are verified to satisfy the conditions of our results, so our theory can be applied to both games. In Section 3 we first recall the proportional $N$-person red-and-black game and then give a counterexample to Pontiggia's conjecture.

\section{Subfair two-person red-and-black game}

Imagine that two players engage in a game and play in stages, each player having an initial fortune of positive integer size. Assume that at each stage each player wants to win the entire fortune of his opponent by betting an amount not greater than his own current fortune. That is to say, both players want to amass the total amount in the system.

Let $M \geq 2$ be the total amount of money in the system and let $S=\{0,1, \ldots, M\}$ be the state space of each player in the game. Once one of the players reaches $M$, the state of neither player can change. We also assume that at each stage each player chooses his action without any knowledge of the action chosen by the other. In fact, all the games we consider are noncooperative in this sense.

If a player always stakes one unit of his current fortune at each stage of the game, we call his strategy a timid strategy. If he always stakes his entire fortune at each stage of the game, we call his strategy a bold strategy.

Denote the two players by I and II. If player I's fortune is $x \in S$, then the action sets are

$$
A_{\mathrm{I}}(x)= \begin{cases}\{1, \ldots, x\} & \text { if } x \in\{1, \ldots, M-1\}, \\ \{0\} & \text { if } x \in\{0, M\},\end{cases}
$$


for player I, and

$$
A_{\mathrm{II}}(x)= \begin{cases}\{1, \ldots, M-x\} & \text { if } x \in\{1, \ldots, M-1\}, \\ \{0\} & \text { if } x \in\{0, M\}\end{cases}
$$

for player II.

Suppose that, at stage $m$, player I has $x^{m}, 1 \leq x^{m} \leq M-1$, units of money and bids an amount $a^{m} \in A_{\mathrm{I}}\left(x^{m}\right)$, while player II bids $b^{m} \in A_{\mathrm{II}}\left(x^{m}\right)$. The law of motion for player I at stage $m$ is defined by

$$
x^{m+1}= \begin{cases}x^{m}+b^{m} & \text { with probability } f\left(\frac{a^{m}}{a^{m}+b^{m}}\right), \\ x^{m}-a^{m} & \text { with probability } 1-f\left(\frac{a^{m}}{a^{m}+b^{m}}\right),\end{cases}
$$

where $f:[0,1] \rightarrow[0,1]$ is an increasing, continuous nonzero (i.e. not everywhere zero) function with $f(0)=0$ and $f(s) \leq s$, called the win probability function of the game. Note that if $x^{m}=0$ or $x^{m}=M$, then the law of motion for player I is defined by $x^{m+1}=x^{m}$ with probability 1 .

Since $f(s) \leq s$, we see that

$$
\begin{aligned}
\mathrm{E}\left[x^{m+1} \mid x^{m}\right] & =\left(x^{m}+b^{m}\right) f\left(\frac{a^{m}}{a^{m}+b^{m}}\right)+\left(x^{m}-a^{m}\right)\left[1-f\left(\frac{a^{m}}{a^{m}+b^{m}}\right)\right] \\
& =x^{m}+\left(a^{m}+b^{m}\right)\left[f\left(\frac{a^{m}}{a^{m}+b^{m}}\right)-\frac{a^{m}}{a^{m}+b^{m}}\right] \\
& \leq x^{m} \quad \text { for } 1 \leq x^{m} \leq M-1,
\end{aligned}
$$

and that $\mathrm{E}\left[x^{m+1} \mid x^{m}\right]=x^{m}$ for $x^{m}=0$ or $x^{m}=M$. Therefore, the process, $\left\{x^{m}\right\}$, of the fortunes of player I is a supermartingale. This means that the game is subfair to player I and superfair to player II. For convenience, this game is called a subfair two-person red-and-black game. As mentioned in the introduction, Pontiggia [3] proposed two types of win probability for player I: (i) $a w /(a+b)$ with $0<w<1$ and (ii) $a w /(a w+b \bar{w})$ with $0<w<\frac{1}{2}$ and $\bar{w}=1-w$, where $a$ and $b$ are the bets of players I and II, respectively. It is easy to verify that these two win probabilities respectively correspond simply to the two probability functions

$$
f_{1}(s)=w s, \quad 0<w<1,
$$

and

$$
f_{2}(s)=\frac{s w}{s w+(1-s) \bar{w}}, \quad 0<w<\frac{1}{2} .
$$

A continuous function $g$ defined on a closed interval $[a, b]$ is said to be convex if, for any $s, t \in[a, b]$ and $\alpha \in[0,1]$, we have $g(\alpha s+(1-\alpha) t) \leq \alpha g(s)+(1-\alpha) g(t)$. It is known from calculus that if $g^{\prime \prime}(s) \geq 0$ for all $s \in(a, b)$, then $g$ is convex on $[a, b]$. The following is a result about a subfair two-person red-and-black game with a convex win probability function.

Theorem 2.1. In a subfair two-person red-and-black game, assume that the win probability function $f$ is a convex function and that player II plays a timid strategy. A bold strategy is then optimal for player $I$. 
Proof. Assume that player II plays a timid strategy. If player I uses a bold strategy, set

$$
Q(x)=\mathrm{P}(\text { player I reaches } M \text { with an initial fortune } x)
$$

The corresponding law of motion at stage $m$ for player I having fortune $x^{m}$ and playing boldly is given by

$$
x^{m+1}=\left\{\begin{array}{ll}
x^{m}+1 & \text { with probability } f\left(\frac{x^{m}}{x^{m}+1}\right), \\
0 & \text { with probability } 1-f\left(\frac{x^{m}}{x^{m}+1}\right),
\end{array} \quad \text { for } 1 \leq x^{m} \leq M-1,\right.
$$

and by $x^{m+1}=x^{m}$ with probability 1 for $x^{m}=0$ or $x^{m}=M$. From this it is not difficult to derive the recursion relation

$$
Q(x)=f\left(r_{x}\right) Q(x+1),
$$

where $r_{x}=x /(x+1)$ and $1 \leq x \leq M-1$. Note that $Q(0)=0$ and $Q(M)=1$.

In order to prove that a bold strategy is optimal for player I when player II plays timidly, it suffices to show that $Q(\cdot)$ is excessive (see Theorem 3.3.10 of [2]) or, equivalently, that the following inequality holds for every $x \in\{1, \ldots, M-1\}$ and every $a \in\{1, \ldots, x\}$ :

$$
f\left(\frac{a}{a+1}\right) Q(x+1)+\left(1-f\left(\frac{a}{a+1}\right)\right) Q(x-a) \leq Q(x) .
$$

Repeatedly using (2.1) yields

$$
Q(x-a)=f\left(r_{x-a}\right) Q(x-a+1)=\cdots=\prod_{i=0}^{a} f\left(r_{x-i}\right) Q(x+1) .
$$

Therefore, (2.2) becomes

$$
f\left(r_{a}\right) Q(x+1)+\left(1-f\left(r_{a}\right)\right) \prod_{i=0}^{a} f\left(r_{x-i}\right) Q(x+1) \leq f\left(r_{x}\right) Q(x+1),
$$

which is equivalent to

$$
f\left(r_{a}\right)+\left(1-f\left(r_{a}\right)\right) \prod_{i=0}^{a} f\left(r_{x-i}\right) \leq f\left(r_{x}\right)
$$

since $Q(x+1)>0$. It is clear that $0<r_{t} \leq r_{x}<1$ if $1 \leq t \leq x$. Now, since $f$ is convex on $[0,1], f(0)=0$, and $r_{t}=\left(r_{t} / r_{x}\right) r_{x}+\left(1-r_{t} / r_{x}\right) \times 0$, we see that

$$
f\left(r_{t}\right) \leq \frac{r_{t}}{r_{x}} f\left(r_{x}\right)+\left(1-\frac{r_{t}}{r_{x}}\right) f(0)=\frac{r_{t}}{r_{x}} f\left(r_{x}\right)
$$


for all $t, 1 \leq t \leq x$. Thus, we have

$$
\begin{aligned}
f\left(r_{a}\right) & +\left(1-f\left(r_{a}\right)\right) \prod_{i=0}^{a} f\left(r_{x-i}\right) \\
& \leq f\left(r_{a}\right)+\left(1-f\left(r_{a}\right)\right) \prod_{i=0}^{a}\left(\frac{r_{x-i}}{r_{x}} f\left(r_{x}\right)\right) \\
& =f\left(r_{a}\right)\left[1-\prod_{i=0}^{a}\left(\frac{r_{x-i}}{r_{x}} f\left(r_{x}\right)\right)\right]+\prod_{i=0}^{a}\left(\frac{r_{x-i}}{r_{x}} f\left(r_{x}\right)\right) \\
& =f\left(r_{a}\right)\left[1-f\left(r_{x}\right)\left(\frac{f\left(r_{x}\right)}{r_{x}}\right)^{a} \frac{x-a}{x}\right]+f\left(r_{x}\right)\left(\frac{f\left(r_{x}\right)}{r_{x}}\right)^{a} \frac{x-a}{x} \\
& \leq \frac{r_{a}}{r_{x}} f\left(r_{x}\right)\left[1-f\left(r_{x}\right)\left(\frac{f\left(r_{x}\right)}{r_{x}}\right)^{a} \frac{x-a}{x}\right]+f\left(r_{x}\right)\left(\frac{f\left(r_{x}\right)}{r_{x}}\right)^{a} \frac{x-a}{x} \\
& =f\left(r_{x}\right)\left[\frac{r_{a}}{r_{x}}-\frac{r_{a}}{r_{x}} f\left(r_{x}\right)\left(\frac{f\left(r_{x}\right)}{r_{x}}\right)^{a} \frac{x-a}{x}+\left(\frac{f\left(r_{x}\right)}{r_{x}}\right)^{a} \frac{x-a}{x}\right] \\
& =f\left(r_{x}\right)\left[\frac{r_{a}}{r_{x}}+\left[1-\frac{r_{a}}{r_{x}} f\left(r_{x}\right)\right]\left(\frac{f\left(r_{x}\right)}{r_{x}}\right)^{a} \frac{x-a}{x}\right] .
\end{aligned}
$$

Let

$$
g(s)=\frac{r_{a}}{r_{x}}+\left(1-\frac{r_{a}}{r_{x}} s\right)\left(\frac{s}{r_{x}}\right)^{a} \frac{x-a}{x} .
$$

Then

$$
g^{\prime}(s)=\frac{a\left(r_{x}-s\right) s^{a-1}}{r_{x}^{a+1}} \frac{x-a}{x} .
$$

This implies that $g^{\prime}(s) \geq 0$ if $0 \leq s \leq r_{x}$. Therefore, $g(s)$ is increasing on $\left[0, r_{x}\right]$. Since $f(s) \leq s$ for all $s \in[0,1]$, we have $f\left(r_{x}\right) \leq r_{x}$. Thus, $g\left(f\left(r_{x}\right)\right) \leq g\left(r_{x}\right)=1$, that is,

$$
\frac{r_{a}}{r_{x}}+\left[1-\frac{r_{a}}{r_{x}} f\left(r_{x}\right)\right]\left(\frac{f\left(r_{x}\right)}{r_{x}}\right)^{a} \frac{x-a}{x} \leq 1 .
$$

Hence,

$$
f\left(r_{a}\right)+\left(1-f\left(r_{a}\right)\right) \prod_{i=0}^{a} f\left(r_{x-i}\right) \leq f\left(r_{x}\right)
$$

and, so, (2.2) holds.

Remark 2.1. Suppose that the win probability function $f$ satisfies $f(s)<s$ for all $s \in(0,1)$ and that player II plays timidly. Then it can be proved that (2.2) is actually an equality if and only if $a=x$. This means that, in this case, the bold strategy is the unique optimal strategy for player I when player II plays timidly.

In the following example we verify the convexity of the win probability functions in the cases proposed by Pontiggia [3].

Example 2.1. (a) In the weighted two-person red-and-black game, the probability of player I winning at stage $m$ is $a^{m} w /\left(a^{m}+b^{m}\right)$, where $0<w<1$. Therefore, the win probability function is $f(s)=s w$ with $0<w<1$. It is clear that $f(s)<s$ for all $s \in(0,1)$. Since 
$f(s)=s w$ is a convex function, it follows from Theorem 2.1 and Remark 2.1 that if player II plays a timid strategy then a bold strategy is the unique optimal strategy for player I.

(b) In the proportional two-person red-and-black game, the win probability for player I at stage $m$ is

$$
\frac{a^{m} w}{a^{m} w+b^{m}(1-w)}, \quad \text { where } 0<w<\frac{1}{2} .
$$

Therefore, the win probability function is $f(s)=s w /[s w+(1-s) \bar{w}]$ with $0<w<\frac{1}{2}$ and $\bar{w}=1-w$. It can be proved that $f(s)<s$. Since, for all $s \in[0,1]$,

$$
f^{\prime \prime}(s)=\frac{2 w \bar{w}(1-2 w)}{[s w+(1-s) \bar{w}]^{3}}>0,
$$

$f$ is a convex function. From Theorem 2.1 and Remark 2.1 it follows that if player II plays a timid strategy then a bold strategy is the unique optimal strategy for player I.

Example 2.2. In a two-person red-and-black game, assume that the win probability function is $f(s)=s^{p}$ for some $p \geq 1$. It is clear that $f$ is a convex function. By Theorem 2.1, a bold strategy is thus optimal for player I when player II plays timidly.

Next we place a condition on the win probability function which ensures that a timid strategy is optimal for player II when player I plays boldly.

Theorem 2.2. In a subfair two-person red-and-black game, assume that player I plays a bold strategy. If $f(s) f(t) \leq f(s t)$ then a timid strategy is optimal for player II.

Proof. Assume that player I plays a bold strategy. If player II adopts a timid strategy then let

$$
T(x)=\mathrm{P}(\text { player II reaches } M \text { with an initial fortune } M-x) .
$$

Recall the function $Q(x)$ defined in the proof of Theorem 2.1: we have $T(x)=1-Q(x)$. If at stage $m$ player II bids an amount $b$, then the corresponding law of motion for player I with $x^{m}$ units is given by

$$
x^{m+1}=\left\{\begin{array}{ll}
x^{m}+b & \text { with probability } f\left(\frac{x^{m}}{x^{m}+b}\right), \\
0 & \text { with probability } 1-f\left(\frac{x^{m}}{x^{m}+b}\right),
\end{array} \quad \text { for } 1 \leq x^{m} \leq M-1,\right.
$$

and by $x^{m+1}=x^{m}$ with probability 1 for $x^{m}=0$ or $x^{m}=M$. As in the proof of Theorem 2.1, it suffices to prove that $T(\cdot)$ is excessive or, equivalently, that the following inequality holds:

$$
f\left(\frac{x}{x+b}\right) T(x+b)+\left(1-f\left(\frac{x}{x+b}\right)\right) T(0) \leq T(x) .
$$

Since $T(x)=1-Q(x)$ and $T(0)=1,(2.3)$ becomes

$$
Q(x) \leq f\left(\frac{x}{x+b}\right) Q(x+b) .
$$


Recall that $r_{x}=x /(x+1)$, so $x /(x+b)=\prod_{i=0}^{b-1} r_{x+i}$ and $Q(x)=Q(x+b) \prod_{i=0}^{b-1} f\left(r_{x+i}\right)$. Since $f(s) f(t) \leq f(s t)$, we have $\prod_{i=0}^{b-1} f\left(r_{x+i}\right) \leq f\left(\prod_{i=0}^{b-1} r_{x+i}\right)$. Therefore,

$$
Q(x)=Q(x+b) \prod_{i=0}^{b-1} f\left(r_{x+i}\right) \leq Q(x+b) f\left(\prod_{i=0}^{b-1} r_{x+i}\right)=Q(x+b) f\left(\frac{x}{x+b}\right) .
$$

Inequality (2.4) and, hence, inequality (2.3) thus hold, and the proof is complete.

Remark 2.2. Suppose that the win probability function $f$ satisfies $f(s) f(t)<f(s t)$ for all $s, t \in(0,1)$ and that player I plays boldly. Then we can prove that $(2.3)$ is actually an equality if and only if $b=1$. This means that, in this case, the timid strategy is the unique optimal strategy for player II when player I plays boldly.

In the following example we verify that both win probability functions proposed by Pontiggia [3] satisfy $f(s) f(t) \leq f(s t)$.

Example 2.3. (a) In the weighted two-person red-and-black game, the win probability function is $f(s)=s w$, where $0<w<1$. Since $f(s) f(t)=s t w^{2}<s t w=f(s t)$ for all $s, t \in(0,1)$, it follows from Theorem 2.2 and Remark 2.2 that if player I plays a bold strategy then a timid strategy is the unique optimal strategy for player II.

(b) In the proportional two-person red-and-black game, the win probability function is $f(s)=$ $s w /[s w+(1-s) \bar{w}]$, where $0<w<\frac{1}{2}$ and $\bar{w}=1-w$. Since, for all $s, t \in(0,1)$,

$$
\begin{aligned}
f(s) f(t)-f(s t) & =\frac{s w}{s w+(1-s) \bar{w}} \frac{t w}{t w+(1-t) \bar{w}}-\frac{s t w}{s t w+(1-s t) \bar{w}} \\
& =\frac{-s t w \bar{w}(t-1)(s-1)(1-2 w)}{[s w+(1-s) \bar{w}][t w+(1-t) \bar{w}][s t w+(1-s t) \bar{w}]} \\
& <0,
\end{aligned}
$$

we have $f(s) f(t)<f(s t)$ for all $s, t \in(0,1)$. From Theorem 2.2 and Remark 2.2 it follows that if player I plays a bold strategy then a timid strategy is the unique optimal strategy for player II.

Example 2.4. In a two-person red-and-black game, assume that the win probability function is $f(s)=s^{p}$, where $p \geq 1$. Since $f(s) f(t)=s^{p} t^{p}=f(s t)$, by Theorem 2.2 a timid strategy is optimal for player II when player I plays boldly.

In a subfair two-person red-and-black game, suppose that the win probability function $f$ is convex and satisfies $f(s) f(t) \leq f(s t)$. From Theorem 2.1 it follows that a bold strategy is optimal for player I if player II plays timidly; moreover, by Theorem 2.2, a timid strategy is optimal for player II if player I plays boldly. Therefore, it is Nash for player I to play a bold strategy and for player II to play a timid strategy; it is usual to say that the profile (bold, timid) is a Nash equilibrium for this game. Hence, we have the following theorem.

Theorem 2.3. In a subfair two-person red-and-black game, assume that the win probability function $f$ is convex and satisfies $f(s) f(t) \leq f(s t)$. Then the profile (bold, timid) is a Nash equilibrium for this game. If, in addition, $f(s)<s$ and $f(s) f(t)<f(s t)$ hold for all $s, t \in(0,1)$, then the profile (bold, timid) is the unique Nash equilibrium for this game. 
Proof. Because the first part of this theorem has been proved above, we need only prove the second part. If $f(s)<s$ and $f(s) f(t)<f(s t)$ hold for all $s, t \in(0,1)$, then, from Remarks 2.1 and 2.2, we can see that the bold strategy is the unique optimal strategy for player I if player II plays timidly, and that the timid strategy is the unique optimal strategy for player II if player I plays boldly. From Lemma A.1 of [3], it follows that the profile (bold, timid) is the unique Nash equilibrium for this game.

Let $f$ and $g$ be win probability functions. Then $f \circ g$ is also a win probability function, since $f \circ g(0)=f(0)=0, f \circ g(x) \leq g(x) \leq x$, and $f \circ g$ is still increasing. We have the following interesting and useful result about this new win probability function $f \circ g$.

Proposition 2.1. Let $f$ and $g$ be two win probability functions. Suppose that $f$ and $g$ are both convex and satisfy $f(s) f(t) \leq f(s t)$ and $g(s) g(t) \leq g(s t)$. In a subfair two-person red-and-black game, take $f \circ g$ as the win probability function. The profile (bold, timid) is then a Nash equilibrium for this game.

Proof. Since $g$ is convex, we have $g(\alpha s+\bar{\alpha} t) \leq \alpha g(s)+\bar{\alpha} g(t)$ for any $s, t, \alpha \in[0,1]$, where $\bar{\alpha}=1-\alpha$. Moreover, since $f$ is increasing and convex, we have

$$
f(g(\alpha s+\bar{\alpha} t)) \leq f(\alpha g(s)+\bar{\alpha} g(t)) \leq \alpha f(g(s))+\bar{\alpha} f(g(t)),
$$

which implies that $f \circ g$ is also convex. On the other hand, for any $s, t \in[0,1]$, since $g(s) g(t) \leq$ $g(s t)$ and $f$ is increasing, we have $f(g(s) g(t)) \leq f(g(s t))$. Since $f(u) f(v) \leq f(u v)$ for any $u, v \in[0,1]$, we have $f(g(s)) f(g(t)) \leq f(g(s) g(t))$. Therefore,

$$
f(g(s)) f(g(t)) \leq f(g(s) g(t)) \leq f(g(s t)),
$$

which means that $f \circ g(s) f \circ g(t) \leq f \circ g(s t)$ for all $s, t \in[0,1]$. The result now follows from Theorem 2.3.

Let $f(s)=s w /[s w+(1-s)(1-w)]$ and $g(s)=s^{p}$, where $0<w<\frac{1}{2}$ and $p \geq 1$. From Proposition 2.1 and Examples 2.1-2.4, we can conclude that, in a subfair two-person red-and-black game, if $f \circ g(s)=s^{p} w /\left[s^{p} w+\left(1-s^{p}\right)(1-w)\right]$ is the win probability function then the profile (bold, timid) is a Nash equilibrium for this game. In fact, we can prove that $f \circ g(s)<s$ and $f \circ g(s) f \circ g(t)<f \circ g(s t)$ hold for all $s, t \in(0,1)$. Hence, by Theorem 2.3, the profile (bold, timid) is the unique Nash equilibrium for this game.

\section{A counterexample}

Consider the proportional $N$-person red-and-black game proposed by Pontiggia [3]. Denote the $N$ players by $1, \ldots, N$. Let $M \geq 2$ be the fixed, total amount of money in the system. The state space of this game is given by the set of all possible $N$-tuples of the fortunes of the $N$ players. Each player wants to reach the goal $M$.

Suppose that at stage $m$ player $j$ has an amount of money $x_{j}^{m}$, for all $j=1, \ldots, N$, with $\sum_{j=1}^{N} x_{j}^{m}=M$. He wants to reach the goal $M$ by betting an amount $a_{j}^{m} \in A_{j}\left(x^{m}\right)$, where $A_{j}(\cdot)$ is the action set for player $j$, defined by

$$
A_{j}\left(x_{j}^{m}\right)= \begin{cases}\left\{1, \ldots, x_{j}^{m}\right\} & \text { if } x_{j}^{m} \in\{1, \ldots, M-1\}, \\ \{0\} & \text { if } x_{j}^{m} \in\{0, M\} .\end{cases}
$$

For each player $j$, define the corresponding weight at stage one by $w_{j}^{1} \in(0,1)$, with $\sum_{j=1}^{N} w_{j}^{1}=1$. During the game, one or more players can go broke, which means that they are 
out of the game. Hence, for each player $j$, recursively define the corresponding weight at stage $m \geq 2$ by

$$
w_{j}^{m}= \begin{cases}0 & \text { if } x_{j}^{m}=0, \\ \frac{w_{j}^{m-1}}{\sum_{\left\{i: x_{i}^{m}>0\right\}} w_{i}^{m-1}} & \text { if } x_{j}^{m}>0,\end{cases}
$$

such that $0 \leq w_{j}^{m}<1$ and $\sum_{j=1}^{N} w_{j}^{m}=1$. The law of motion for player $j$ with $x_{j}^{m}$ units at stage $m$ is defined by

$$
x_{j}^{m+1}= \begin{cases}x_{j}^{m}-a_{j}^{m}+\sum_{i=1}^{N} a_{i}^{m} & \text { with probability } \frac{a_{j}^{m} w_{j}^{m}}{\sum_{i=1}^{N} a_{i}^{m} w_{i}^{m}}, \\ x_{j}^{m}-a_{j}^{m} & \text { with probability } 1-\frac{a_{j}^{m} w_{j}^{m}}{\sum_{i=1}^{N} a_{i}^{m} w_{i}^{m}},\end{cases}
$$

and by $x_{j}^{m+1}=x_{j}^{m}$ with probability 1 for $x_{j}^{m}=0$ or $x_{j}^{m}=M$.

For the above proportional $N$-person red-and-black game, Pontiggia proposed the following conjecture (slightly modified from the original).

Conjecture 3.1. ([3].) Let $\sigma$ be the following strategy. Suppose that there are $k^{m}, 2 \leq k^{m} \leq$ $N$, players remaining in the game at stage $m$. For an active player (say player $j$ ), if the corresponding weight, $w_{j}^{m}$, is less than or equal to $1 / k^{m}$, then he bids his entire fortune; otherwise, he bids one unit of it.

It is optimal for each player to use strategy $\sigma$ when the other players all use strategy $\sigma$.

The following is a counterexample to this conjecture.

Counterexample 3.1. In a proportional three-person red-and-black game, let $M=7$ be the total amount of money in the system. Suppose that at stage $m$ player $j$ has $x_{j}^{m}$ units of money and bids an amount $a_{j}^{m} \in\left\{1, \ldots, x_{j}^{m}\right\}$ if $x_{j}^{m}>0$ and an amount $a_{j}^{m}=0$ otherwise, for all $j=1,2,3$. Let $\left(w_{1}^{1}, w_{2}^{1}, w_{3}^{1}\right)=(w, \bar{w} / 2, \bar{w} / 2)$, where $0<w \leq \frac{1}{6}$ and $\bar{w}=1-w$. If no one goes broke at stage $m$, then $\left(w_{1}^{m}, w_{2}^{m}, w_{3}^{m}\right)=(w, \bar{w} / 2, \bar{w} / 2)$ and $w_{1}^{m}<\frac{1}{3}<w_{2}^{m}=w_{3}^{m}$; in this case player 1 is subfair and players 2 and 3 are superfair. If either player 2 or player 3 goes broke at stage $m$, then $w_{1}^{m}=2 w /(1+w)<\frac{1}{2}$ and either $w_{2}^{m}=(1-w) /(1+w)>\frac{1}{2}$ or $w_{3}^{m}=(1-w) /(1+w)>\frac{1}{2}$; in this case player 1 is subfair and the other remaining player is superfair. So, under strategy $\sigma$, player 1 always plays boldly and players 2 and 3 always play timidly.

Let $x, y$, and $z$ be any nonnegative integers with $x+y+z=7$. If players 1,2 , and 3 have $x, y$, and $z$ as their respective initial fortunes and all adopt strategy $\sigma$, let $Q(x, y, z)$ denote the probability that player 1 reaches the amount 7 . If at stage $m$ no one goes broke, then under strategy $\sigma$ the corresponding law of motion for player 1 is

$$
x_{1}^{m+1}=\left\{\begin{array}{ll}
x_{1}^{m}+2 & \text { with probability } \frac{x_{1}^{m} w}{x_{1}^{m} w+\bar{w}}, \\
0 & \text { with probability } \frac{\bar{w}}{x_{1}^{m} w+\bar{w}},
\end{array} \text { for } 1 \leq x_{1}^{m} \leq M-1,\right.
$$


and is $x_{1}^{m+1}=x_{1}^{m}$ with probability 1 for $x_{1}^{m}=0$ or $x_{1}^{m}=M$. If at stage $m$ either player 2 or player 3 goes broke, then under strategy $\sigma$ the corresponding law of motion for player 1 is

$$
x_{1}^{m+1}=\left\{\begin{array}{ll}
x_{1}^{m}+1 & \text { with probability } \frac{2 x_{1}^{m} w}{2 x_{1}^{m} w+\bar{w}}, \\
0 & \text { with probability } \frac{\bar{w}}{2 x_{1}^{m} w+\bar{w}},
\end{array} \text { for } 1 \leq x_{1}^{m} \leq M-1\right.
$$

and is $x_{1}^{m+1}=x_{1}^{m}$ with probability 1 for $x_{1}^{m}=0$ or $x_{1}^{m}=M$. Therefore, for $x y z \neq 0$,

$$
Q(x, y, z)=\frac{x w}{x w+\bar{w}} Q(x+2, y-1, z-1)
$$

and

$$
Q(x, y, 0)=\frac{2 x w}{2 x w+\bar{w}} Q(x+1, y-1,0) .
$$

To show that Pontiggia's conjecture does not hold, we now show that if the value of $w$ is small enough, then the bold strategy is not optimal for player 1 when players 2 and 3 play timidly. Assume that initially player 1 has two units, player 2 has four units, and player 3 has one unit. Then the probability that player 1 reaches the amount 7 by playing boldly is $Q(2,4,1)$ when players 2 and 3 play timidly.

Let $\beta$ be the strategy according to which a player bets one unit at the first stage and plays boldly thereafter. Let $Q_{\beta}$ denote the probability that player 1 adopts strategy $\beta$ to reach the total amount 7 when players 2 and 3 play timidly. Then

$$
Q_{\beta}=\frac{w}{w+\bar{w}} Q(4,3,0)+\frac{\bar{w} / 2}{w+\bar{w}} Q(1,6,0)+\frac{\bar{w} / 2}{w+\bar{w}} Q(1,3,3) .
$$

In the following, we will prove that $Q_{\beta}>Q(2,4,1)$ for sufficiently small values of $w$. Repeatedly applying (3.2) yields

$$
\begin{aligned}
Q(1,6,0) & =\frac{2 w}{2 w+\bar{w}} Q(2,5,0), & Q(2,5,0) & =\frac{4 w}{4 w+\bar{w}} Q(3,4,0), \\
Q(3,4,0) & =\frac{6 w}{6 w+\bar{w}} Q(4,3,0), & Q(4,3,0) & =\frac{8 w}{8 w+\bar{w}} Q(5,2,0), \\
Q(5,2,0) & =\frac{10 w}{10 w+\bar{w}} Q(6,1,0), & Q(6,1,0) & =\frac{12 w}{12 w+\bar{w}} Q(7,0,0) .
\end{aligned}
$$

Applying (3.1) yields

$$
\begin{array}{ll}
Q(2,4,1)=\frac{2 w}{2 w+\bar{w}} Q(4,3,0), & Q(1,3,3)=\frac{w}{w+\bar{w}} Q(3,2,2), \\
Q(3,2,2)=\frac{3 w}{3 w+\bar{w}} Q(5,1,1), & Q(5,1,1)=\frac{5 w}{5 w+\bar{w}} Q(7,0,0) .
\end{array}
$$


Since $Q(7,0,0)=1$, we have

$$
\begin{aligned}
& Q(4,3,0)=\frac{960 w^{3}}{\prod_{i=4}^{6}(2 i w+\bar{w})}=O\left(w^{3}\right), \\
& Q(1,6,0)=\frac{46080 w^{6}}{\prod_{i=1}^{6}(2 i w+\bar{w})}=O\left(w^{6}\right), \\
& Q(1,3,3)=\frac{15 w^{3}}{\prod_{i=0}^{2}[(2 i+1) w+\bar{w}]}=O\left(w^{3}\right)
\end{aligned}
$$

as $w \rightarrow 0$. It follows that $Q(2,4,1)=[2 w /(2 w+\bar{w})] Q(4,3,0)=O\left(w^{4}\right)$ and $Q_{\beta}=O\left(w^{3}\right)$ as $w \rightarrow 0$. Thus, $Q_{\beta}-Q(2,4,1)>0$ for sufficiently small values of $w$. Therefore, for player 1 , the strategy $\beta$ is better than the strategy $\sigma$ for such values of $w$. Hence, Pontiggia's conjecture does not hold.

Remark 3.1. In this case, a detailed calculation shows that, for sufficiently small values of $w$, the strategy $\beta$ is indeed the optimal strategy for player 1 when players 2 and 3 play timidly.

\section{Acknowledgement}

The authors wish to thank the referee for his/her helpful suggestions, which improved the paper and made it more readable.

\section{References}

[1] Dubins, L. E. And Savage, L. J. (1976). Inequalities for Stochastic Processes: How to Gamble if You Must, 2nd edn. Dover, New York.

[2] Maitra, A. P. and Sudderth, W. D. (1996). Discrete Gambling and Stochastic Games. Springer, New York.

[3] Pontiggia, L. (2005). Two-person red-and-black with bet-dependent win probability. Adv. Appl. Prob. 37, 75-89.

[4] Ross, S. M. (1974). Dynamic programming and gambling models. Adv. Appl. Prob. 6, 598-606.

[5] SeCCHI, P. (1997). Two-person red-and-black stochastic games. J. Appl. Prob. 34, 107-126. 